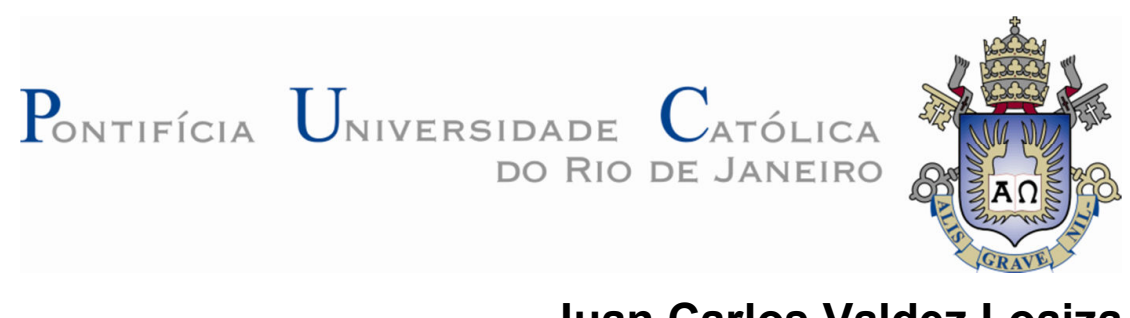

Juan Carlos Valdez Loaiza

Ignição por Compressão com Reatividade Controlada de Óleo Diesel e Etanol em Máquina de Compressão Rápida

Tese de Doutorado

Tese apresentada ao Programa de Pós-Graduação em Engenharia Mecânica da PUC-Rio como requisito parcial para obtenção do título de Doutor Engenharia Mecânica.

Orientador: Prof. Sergio Leal Braga

Rio de Janeiro

Agosto de 2014 


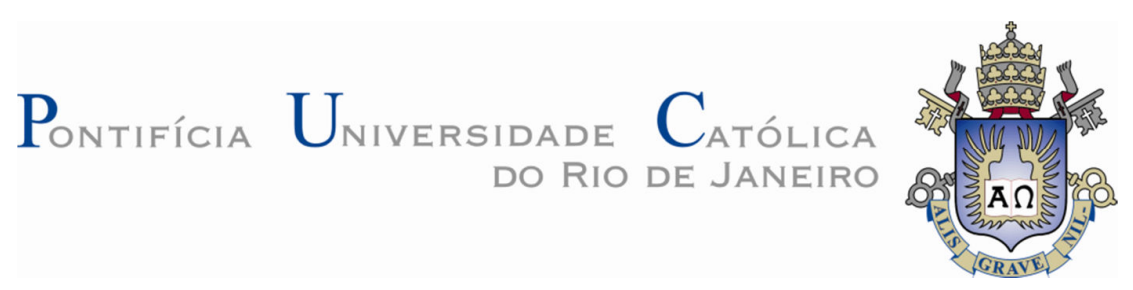

Juan Carlos Valdez Loaiza

\section{Ignição por Compressão com Reatividade Controlada de Óleo Diesel e Etanol em Máquina de Compressão Rápida}

Tese apresentada como requisito parcial para obtenção do grau de Doutor pelo Programa de Pósgraduação em Engenharia Mecânica do Departamento de Engenharia Mecânica do Centro Técnico Científico da PUC-Rio. Aprovada pela Comissão Examinadora abaixo assinada.

Prof. Sergio Leal Braga Orientador

Pontifícia Universidade Católica do Rio de janeiro

Prof. Carlos Valois Maciel Braga Pontifícia Universidade Católica do Rio de Janeiro

Prof. Marcos Sebastião de Paula Gomes Pontifícia Universidade Católica do Rio de Janeiro

Tadeu Cavalcante Cordeiro de Melo Centro de Pesquisas da PETROBRAS

Prof. Wladimyr Mattos da Costa Dourado Instituto de Aeronáutica e Espaço

Ricardo Hernandez Pereira

Prof José Eugenio Leal Coordenador Setorial do Centro Técnico Cientifico - PUC-Rio

Rio de Janeiro, 11 de Agosto de 2014 
Todos os direitos reservados. É proibida a reprodução total ou parcial do trabalho sem autorização da universidade, do autor e do orientador.

\section{Juan Carlos Valdez Loaiza}

Graduou-se em Engenharia Mecânica pela Universidad Católica de Santa Maria, Arequipa-Perú em 2006. Obteve o Título de Mestre em Engenharia Mecânica na PUC-Rio em 2009 (Termociências, numérico, com ênfases na simulação de sistemas e componentes de refrigeração e condicionamento de ar, sistemas secundários e nanofluidos). Atualmente é pesquisador no LEV/PUC-Rio, estando envolvido no uso de combustíveis alternativos em motores de combustão interna.

Ficha Catalográfica

Loaiza, Juan Carlos Valdez

Ignição por compressão com reatividade controlada de óleo diesel e etanol em máquina de compressão rápida / Juan Carlos Valdez Loaiza; orientador: Sérgio Leal Braga. 2014.

134 f. : il. (color.) ; $30 \mathrm{~cm}$

Tese (doutorado)-Pontifícia Universidade Católica do Rio de Janeiro, Departamento de Engenharia Mecânica, 2014.

Inclui bibliografia

1. Engenharia mecânica - Teses. 2. Combustão. 3. Ignição por compressão com reatividade controlada. 4. Dualfuel. 5. Diesel-etanol. 6. Máquina de compressão rápida. I. Braga, Sergio Leal. II. Pontifícia Universidade Católica do Rio de Janeiro. Departamento de Engenharia Mecânica. III. Título.

CDD: 621 
Deixa ir às pessoas que só chegam para compartilhar queixas, problemas, histórias desastrosas, medo e juízo dos outros. Se alguém procura um cubo para jogar seu lixo, procura que não seja tua mente - DALAI LAMA. 


\section{Agradecimentos}

A minha mãe e irmã por seu amor e apoio incondicional.

Ao Professor Sérgio Leal Braga, meu orientador, pelo apoio recebido e pela ideia original do trabalho.

A meus amigos Mijail, Marco, Oberdan, Marvin, Daniela, Veronica, Dario, Javier pela amizade e pelos bons momentos compartilhados nestes anos.

Aos Engenheiros, Giovanni Calfa, Severino Wanderley, Nestor Correa, Fernando Zegarra e Leonardo Costa Braga, pela colaboração, interesse e apoio técnico na realização deste trabalho.

Aos funcionários do ITUC, Marco, Renato, Leandro, Luciano, pelo suporte e apoio.

Aos técnicos do laboratório de Engenharia Veicular, assim como ao estagiário Felipe Murad.

Ao Departamento de Engenharia Mecânica por seu apoio durante todos estes anos.

Agradecimentos em particular para os órgãos de fomento à pesquisa $\mathrm{CNPq}$, CAPES, pelo apoio financeiro, sem o qual este trabalho simplesmente não teria sido possível.

A todos aqueles que contribuíram positivamente para a realização deste trabalho. 


\section{Resumo}

Loaiza, Juan Carlos Valdez; Braga Sergio Leal. Ignição por Compressão com Reatividade Controlada de Óleo Diesel e Etanol em Máquina de Compressão Rápida. Rio de Janeiro, 2014. 134p. Tese de Doutorado Departamento de Engenharia Mecânica, Pontifícia Universidade Católica do Rio de Janeiro.

Serão necessários muitos anos para que os biocombustíveis sejam capazes de substituir integralmente os derivados fósseis. Este trabalho visa estudar formas alternativas de conversão de energia contida nos combustíveis utilizados em motores de combustão interna. Maiores eficiências na conversão da energia contida no combustível e uma menor emissão dos gases de exaustão são benefícios associados à ignição por compressão de reatividade controlada, RCCI, onde dois fluidos com diferentes reatividades são introduzidos na câmara de combustão em instantes diferentes. Optou-se pelo uso de uma máquina de compressão rápida, $\mathrm{MCR}$, capaz de controlar parâmetros relevantes, como taxa de compressão, pressões, tempos de injeção, que foi adaptada para receber dois sistemas de injeção direta na câmara de combustão. Como segundo combustível, que substitui parcialmente o óleo diesel, que é empregado tradicionalmente em motores de ignição por compressão, optou-se pelo etanol hidratado. Os estudos revelaram que diferentes formas de injeção dos dois combustíveis produzem processos muito diferentes, para as mesmas quantidades de combustíveis injetados. Os resultados são apresentados na forma de pressão indicada como função do ângulo equivalente, bem como calor liberado e atraso de ignição. Experiências foram conduzidas para uma ou duas injeções de etanol por ciclo, em diferentes tempos. Altas razões de substituição do combustível fóssil foram obtidas, quando comparadas com a técnica de fumigação, onde o segundo combustível é misturado externamente ao ar de combustão.

\section{Palavras chaves}

Combustão; Ignição por Compressão com Reatividade Controlada; Dual Fuel; Diesel - Etanol; Máquina de Compressão Rápida. 


\section{Abstract}

Loaiza, Juan Carlos Valdez; Braga, Sergio Leal (Advisor). Reactivity Controlled Compression Ignition of Diesel Fuel and Ethanol in Rapid Compression Machine. Rio de Janeiro, 2014. 134p. Tese de Doutorado Departamento de Engenharia Mecânica, Pontifícia Universidade Católica do Rio de Janeiro.

Many years will be needed for biofuels or other renewable sources to be able to fully replace fossil fuels. This work aims to study alternative ways of converting energy contained in fuels used in internal combustion engines. Higher efficiencies in converting the energy contained in the fuel and lower emission of harmful exhaust gases are benefits associated with the Reactivity Controlled Compression Ignition, known for RCCI. In this type of combustion, two fluids with different ignition-reactivity characteristics are introduced into the combustion chamber at different times. To better understand this phenomenon, it was used a $\mathrm{RCM}$, that is able to control, more easily, relevant parameters such as compression ratio, temperatures, pressures, injection times etc. As a second fuel, which partially replaces the diesel, which is traditionally used in compression ignition engines, it was used the ethanol. The RCM was then adapted to receive two systems for direct injection into the combustion chamber. Studies have shown that different forms of injection of the two fuels produce very different processes to the same amount of fuel injected. The results are presented in the form of indicated pressure as a function of position. Heat released and ignition delay are also presented. Experiments were conducted for one or two injections of ethanol per cycle at different times. High substitution rates of the fossil fuel were obtained when compared to injections of external mixtures of diesel and ethanol or fumigation technique, where the second fuel is mixed externally with the combustion air.

\section{Keywords}

Combustion; reactivity controlled compression ignition; dual-fuel; dieselethanol; rapid compression machine. 


\section{Sumário}

1. Introdução 19

1.1. Objetivo 24

1.2. Justificativa 24

1.3. Contexto histórico 25

1.4. Conteúdo do trabalho 30

2. Revisão Bibliográfica 31

2.1 Mapa Razão de Equivalência - Temperatura $(\phi-T)$

2.2. Novos Modos de Combustão em Motores $\mathrm{Cl} 37$

2.2.1 Ignição por Compressão de Carga Homogênea 37

2.2.2 ignição por Compressão de Carga Pré-misturada 38

2.2.3 Combustão em Baixa Temperatura 39

2.2.4 Ignição por Compressão de Reatividade Controlada 41

2.3 Estratégias de injeção de combustivel 45

2.4 Estratégias de injeção Dual Fuel 50

2.4.1 Fumigação $\quad 51$

2.4.2 Dupla Injeção Direta 56

3. Aparato e Procedimento Experimental 60

3.1 Máquina de Compressão Rápida 60

3.2 Princípo de Funcionamento da Máquina de Compressão Rápida 62

3.3 Configuração Dupla Injeção Direta 69

3.4 Equipamentos Complementários 73

3.5 Calibração dos Injetores para Óleo Diesel e Etanol 75

3.6 Procedimento Experimental $\quad 77$

3.7 Cálculo da Quantidade de Combustível Injetada 78

3.8 Tempo de Compressão $\quad 80$

3.9 Velocidade Média do Pistão 80

3.10 Calor Total Liberado $\quad 81$

3.11 Vazão Mássica 82 
4 Desenvolvimento dos Ensaios e Resultados 83

4.1 Etapa I - Caso Base - Diesel 84

4.2 Etapa II - Dual Fuel - Injeção Simples 90

4.3 Etapa III - Dual Fuel - Injeção Dupla de Etanol 94

5 Conclusões e Sugestões para Trabalhos Futuros 114

5.1 Conclusões 114

$\begin{array}{ll}5.2 \text { Sugestões para Trabalhos Futuros } & 115\end{array}$

6 Referências Bibliográficas 117

Anexo I: Dados Técnicos do Sensor de Pressão Kistler tipo 2852A 129 Anexo II: iPOD 8427

Anexo III: High Pressure Regulator (HPR) 8244

Anexo IV: Propriedades Termofísicas 132

$\begin{array}{ll}\text { Anexo V: Análise de incertezas experimentais } & 133\end{array}$ 


\section{Lista de figuras}

Figura 1 - Análises do ciclo Diesel em um motor ICO com diesel e Etanol 22

Figura 2 - Cotação do Barril de Petróleo: 1970 - 200528

Figura 3 - Evolução cronológica da legislação sobre poluentes Nos EUA em veículos de passageiros com motores ICO 29

Figura 4 - Evolução tecnologias dos motores ICO 30

Figura 5 - Emissão de CO2 por categoria de veiculo 30

Figura 6 - Primeira representação gráfica do mapa $\phi$ - T 33

Figura 7 - Mapa $(\phi-T)$ pressão constante de $6 \mathrm{MPa}$ e tempo de reação de $1 \mathrm{~ms}$

Figura 8 - Mapa $(\phi-T)$ com tempo de reação $2 m s$ representando regiões de formação de MP e NOx

Figura 9 - Monóxido de Carbono (CO) como uma função da temperatura e a razão de equivalência 36

Figura 10 - Hidrocarbonetos não queimados (HC) como uma função da temperatura e a razão de equivalência

Figura 11 - Alta eficiência e regimes de combustão limpa sobre um mapa $\phi-T$

Figura 12 - Modos de Combustão com alta eficiência e regimes de combustão limpa sobre um mapa $\phi$ - $T$

Figura 13 - Esquema das diversas estratégias de combustão

LTC segundo a distância na qual acontece a injeção de

Combustível

Figura 14 - Efeito sobre as emissões dos modos de combustão $\mathrm{CDC}, \mathrm{PCCl}$ e RCCl

Figura 15 - Avanços das estratégias de combustão convergindo tanto em Hardware quanto em combustível

Figura 16 - Pressão do cilindro, para diferentes Modos de Combustão 44

Figura 17 - Classificação dos conceitos de combustão em fase pré-misturada segundo o método de injeção do combustível

Figura 18 - Estratégia de injeção de massa vs o tempo para as 
tipologias básicas de injeção múltipla: (a)estratégia de injeção piloto,

(b) estratégia de pós injeção e (c) estratégia de injeção partida

Figura 19 - Esquema dos diferentes parâmetros que entram em jogo na hora de definir uma estratégia de injeção múltipla. Neste caso se representa uma estratégia de injeção partida

Figura 20 - Injeções múltiplas.

Figura 21 - Emissão de fumaça (FSN), NOx e consumo de Combustível com uma estratégia de três injeções vs o tempo entre pulsos "Dwell"

Figura 22 - Efeito da múltipla injeção no desempenho e emissões

De um motor de injeção direta com alta velocidade de rotação

Figura 23: Fumigação aplicada ao método RCCl

Figura 24: Motor de quatro cilindros operando no modo Dual Fuel, com injeção indireta de gasolina e injeção direta de diesel, controladas através de uma só UCE

Figura 25 - Esquema do aparato experimental utilizado por

Egusquisa (2011)

Figura 26 - Variação das curvas de pressão com a substituição

Diesel/etanol e a carga do motor (RPM: 1.800); injeção do diesel: $\mathrm{P0}=9^{\circ}$ APMS.

Figura 27 - Diagrama do posicionamento da injeção direta Dual Fuel

Figura 28 - Configuração de um injetor central e quatro válvulas em um motor ICO.

Figura 29 - Vista em corte de uma MCR mostrando a parte Interna

Figura 30 - Máquina de Compressão Rápida instalada no

Laboratório de Engenharia Veicular (PUC-Rio)

Figura 31 - Áreas funcionais da Máquina de Compressão Rápida Instalada no Laboratório de Engenharia Veicular PUC-Rio

Figura 32 - Posição do sistema no início do experimento

Figura 33 - Posição do sistema no final do experimento

Figura 34 - Interface de operação da Máquina de Compressão Rápida, software CAMAS

Figura 35 - Interface de operação do Trigger Signais no software 
Figura 36 - Interface do Crank Calculator no software CAMAS

Figura 37 - Diagrama de montagem dos dois injetores na MCR

Figura 38 - Esquema de montagem simplificado do aparato experimental de dupla injeção direta Dual Fuel (Diesel-Etanol)

Figura 39 - Cabeçote do cilindro da MCR mostrando os dois injetores(Diesel-Etanol)

Figura 40 - Dados Geométricos da MCR no modo Dual Fuel

Figura 41 - Montagem dos sistemas de injeção de combustível Dual Fuel na MCR no LEV da PUC-Rio

Figura 42 - Sistema utilizado para pressurizar e controlar a injeção do Etanol

Figura 43 - Tela principal do software Kistler SCP, utilizado para redefinir o valor do sensor de pressão

Figura 44 - Tela principal do software XIPOD, interface com o Injetor de óleo diesel

Figura 45 - Tela principal do software WINHPR, interface com regulador da bomba de alta pressão para o óleo diesel

Figura 46 - Curva de calibração do injetor com etanol hidratado (H100), pressão de injeção 100 bar

Figura 47 - Curva de calibração do injetor do óleo diesel, pressão de injeção 1070 bar

Figura 48 - Pressão vs Ângulo no interior do cilindro para teste com óleo diesel

Figura 49 - Calor vs Ângulo no interior do cilindro para teste com óleo diesel

Figura 50 - Estratégia de injeção Dual Fuel, caso 1

Figura 51 - Estratégia de injeção Dual Fuel, caso 2

Figura 52 - Pressão vs Ângulo no interior do cilindro, cargas de óleo diesel puro y duas estratégias Dual Fuel (35\% Etanol - 25\% Diesel)

Figura 53 - Calor vs Ângulo no interior do cilindro, cargas de óleo diesel puro y duas estratégias Dual Fuel (35\% Etanol - 25\% Diesel)

Figura 54 - Pressão vs Ângulo no interior do cilindro, cargas de óleo diesel puro y duas estratégias Dual Fuel (30\% Etanol - 45\% Diesel)

Figura 55 - Calor vs Ângulo no interior do cilindro, cargas de óleo 
diesel puro y duas estratégias Dual Fuel (30\% Etanol - 45\% Diesel)

Figura 56 - Pressão vs Ângulo no interior do cilindro para teste com óleo diesel, Etapa III

Figura 57 - Calor vs Ângulo no interior do cilindro para teste com óleo diesel, Etapa III

Figura 58 - Imagens da Combustão Diesel, com TC=20:1

Figura 59 - Estratégia de injeção Dual Fuel, caso 1, Etapa III

Figura 60 - Pressão vs Ângulo no interior do cilindro para teste

Dual Fuel (Etanol - Diesel) etapa III

Figura 61 - Vazamento no injetor de Etanol no interior da MCR durante os testes com TC=20:1

Figura 62 - Calor liberado vs Ângulo no interior do cilindro para teste Dual Fuel (Etanol - Diesel) etapa III

Figura 63 - Imagens da combustão Dual Fuel, Diesel Etanol com TC = 20:1 e razão de substituição igual a $80 \%$

Figura 64 - Estratégia de injeção Dual Fuel, três injeções, Etapa III

Figura 65 - Pressão vs Ângulo no interior do cilindro, Estratégias

Dual Fuel etapa III

Figura 66 - Calor vs Ângulo no interior do cilindro, Estratégias Dual Fuel etapa III

Figura 67 - Pressão vs Ângulo no interior do cilindro, influencia da Pós-injeção no atraso da combustão. Estratégias Dual Fuel etapa III Figura 68 - Calor vs Ângulo no interior do cilindro, influencia da pós-injeção no atraso da combustão. Estratégias Dual Fuel etapa III

Figura 69 - Pressão vs Ângulo no interior do cilindro para teste com óleo diesel puro e estratégias Dual Fuel com duas e três injeções mudando o ponto de injeção do óleo diesel

Figura 70 - Calor vs Ângulo no interior do cilindro para teste com óleo diesel puro e estratégias Dual Fuel com duas e três injeções mudando o ponto de injeção do óleo diesel

Figura 71 - Pré-injeção de etanol - Injeção de óleo diesel

Figura 72 - Pós-injeção de etanol - Injeção de óleo diesel

Figura 73 - injeção simultânea óleo diesel - etanol

Figura 74 - Pressão vs Ângulo, mostrando o efeito do ponto de 
injeção do etanol com referência a injeção de óleo diesel

Figura 75 - Calor vs Ângulo, mostrando o efeito do ponto de injeção do etanol com referência a injeção de óleo diesel 


\section{Lista de Tabelas}

Tabela 1 - Etanol como combustível em motores ICO 23

Tabela 2 - Modos de combustão LTC 41

Tabela 3 - Resumo das principais conclusões decorrentes da fumigação de etanol em motores ICO 54

Tabela 4 - Características da MCR modelo TeRCM-k84. 67

Tabela 5 - Características do injetor para etanol hidratado $(\mathrm{H} 100) \quad 76$

Tabela 6 - Especificações do injetor HDEV5 76

Tabela 7 - Características do injetor para óleo diesel. $\quad 77$

Tabela 8 - Especificações do injetor tipo standard LD 78

Tabela 9 - Dados do Motor DW10 CTED4 79

Tabela 10 - Dados do teste na MCR para 100\% da carga máxima. $\quad 79$

Tabela 11 - Resumo das estratégias de injeção Dual Fuel 85

Tabela 12 - Calibração da MCR procurando uma pressão

final $=38,25 \pm 0,25$ bar $\quad 86$

Tabela 13 - Resultado dos testes na MCR com injeção de óleo

diesel, TC=16:1 87

Tabela 14 - Resultado dos testes na MCR com injeção de óleo

diesel, TC=20:1 95

Tabela IV - Propriedades termofísicas do ar, diesel e etanol

$\left(20^{\circ} \mathrm{C}\right.$ e $\left.1 \mathrm{~atm}\right)$ 


\section{Lista de símbolos}

\begin{tabular}{|c|c|c|}
\hline$A$ & $\mathrm{Ar}$ & \\
\hline AAVE & Ângulo de abertura das válvulas de escapamento & \\
\hline APMS & Antes do Ponto Morto Superior & \\
\hline BSU & Unidade de Fumaça Bosch & \\
\hline C & Carbono, Combustível & \\
\hline CAI & Auto Ignição Controlada & \\
\hline CCEU & Ciclo de Condução Extra-Urbano & \\
\hline CDC & Combustão Diesel Convencional & \\
\hline CEC & Consumo Especifico de Combustível & \\
\hline $\mathrm{CO}$ & Monóxido de Carbono & \\
\hline $\mathrm{CO}_{2}$ & Dióxido de Carbono & \\
\hline $\mathrm{Cv}$ & Calor a Volume Constante & \\
\hline dBA & Decibel com filtro tipo A & \\
\hline DCDC & $\begin{array}{l}\text { Combustão Diesel limpa com combustível } \\
\text { oxigenado }\end{array}$ & \\
\hline DF & Dual Fuel & \\
\hline DPF & Filtro de Partículas & \\
\hline DI & Injeção Direta & \\
\hline DME & Di-Methyl-Ether & \\
\hline DOE & Departamento de Energia dos Estados Unidos & \\
\hline DP & Duração de cada Pulso & \\
\hline d & Derivada & \\
\hline EGR & Recirculação de Gases de Exaustão & \\
\hline EURO & Comissão Europeia para veículos leves e pesados & \\
\hline EPA & Agência de Proteção Ambiental & \\
\hline ETBE & Ethyl-Tentiary- Butyl-Ether & \\
\hline EtOH & Etanol & \\
\hline EUA & Estados Unidos de América & \\
\hline EUDC & Ciclo de Condução Extra-Urbano & \\
\hline FMQ & Fração de Massa Queimada & \\
\hline FP & Filtro de Partículas & \\
\hline g & Gramo & \\
\hline GBC & Biogás & \\
\hline GDI & Injeção Direta de Gasolina & \\
\hline GEE & Gases de Efeito Estufa & \\
\hline GNV & Gás Natural Veicular & \\
\hline GNC & Gás Natural Comprimido & \\
\hline GLP & Gás Liquefeito de Petróleo & \\
\hline $\mathrm{H}$ & Hidrogênio & \\
\hline $\mathrm{H} 100$ & etanol hidratado & \\
\hline HiMICS & Sistema Inteligente de Combustão com Carga & \\
\hline & $\begin{array}{l}\text { Homogênea e Múltiplas Injeções } \\
\text { Hidrocarbonetos não queimados }\end{array}$ & \\
\hline $\mathrm{HCCl}$ & $\begin{array}{l}\text { Hidrocarbonetos nao quelmados } \\
\text { Iqnicão por Compressão de Carqa Homogênea }\end{array}$ & \\
\hline HCDC & Combustão Diesel com Carga Homogênea & \\
\hline $\mathrm{HCLI}$ & Injeção Atrasada com Carga Homogênea & \\
\hline
\end{tabular}




\begin{tabular}{|c|c|c|}
\hline HDDI & $\begin{array}{l}\text { Motores de cilindrada unitária elevada dedicados } \\
\text { a Transporte de mercadorias }\end{array}$ & {$[-]$} \\
\hline HPLI & Injeção Atrasada Altamente Pré-misturada & {$[-]$} \\
\hline HSDI & $\begin{array}{l}\text { Motores de cilindrada unitária pequena e injeção } \\
\text { direta dedicados ao transporte de passageiros }\end{array}$ & {$[-]$} \\
\hline ICE & Ignição por Centelha & {$[-]$} \\
\hline ICO & Ignição por Compressão & {$[-]$} \\
\hline IEA & Agência Internacional de Energia & {$[-]$} \\
\hline ISAF & Simpósio Internacional de Álcool Combustível & {$[-]$} \\
\hline IVO & Ângulo de fechamento das válvulas de admissão & {$[-]$} \\
\hline kW & Kilowatt & {$[-]$} \\
\hline $\mathrm{kg}$ & kilogramo & {$[-]$} \\
\hline $\mathrm{L}$ & litro & [l] \\
\hline LEV & Laboratório de Engenharia Veicular & {$[-]$} \\
\hline LTC & Combustão em baixa temperatura & {$[-]$} \\
\hline $\mathrm{m}$ & massa & {$[\mathrm{kg}, \mathrm{g}]$} \\
\hline $\mathrm{MCl}$ & Motor de Combustão Interna & {$[-]$} \\
\hline MCR & Máquina de Compressão Rápida & {$[-]$} \\
\hline MK & Cinética Modulada & {$[-]$} \\
\hline MTBE & Methyl-Tertiary-Butyl Ether & {$[-]$} \\
\hline MP & Material Particulado & {$[-]$} \\
\hline $\begin{array}{l}\mathrm{MPa} \\
\mathrm{ms}\end{array}$ & $\begin{array}{l}\text { Megapascal } \\
\text { milisegundo }\end{array}$ & $\begin{array}{l}{[\mathrm{MPa}]} \\
{[\mathrm{ms}]}\end{array}$ \\
\hline MULDIC & Combustão Diesel de Fases Múltiplas & {$[-]$} \\
\hline MWM & Motor Werke Mannheim - Motores Diesel LTda & {$[-]$} \\
\hline NADI & Injeção Direta em Ângulo & {$[-]$} \\
\hline NEDC & Novo Ciclo de Condução Europeu & {$[-]$} \\
\hline $\mathrm{NO}_{\mathrm{x}}$ & Todos os Óxidos de Nitrogênio & {$[-]$} \\
\hline NTC & Coeficiente de Temperatura Negativa & {$[-]$} \\
\hline $\mathrm{N}$ & Rotação do motor & [RPM] \\
\hline $\mathrm{N}_{2}$ & Nitrogênio & {$[-]$} \\
\hline $\mathrm{O}_{2}$ & Oxigênio & {$[-]$} \\
\hline OPEP & Organização dos Países Exportadores de Petróleo & {$[-]$} \\
\hline ORNL & Oak Ridge National Laboratory & {$[-]$} \\
\hline $\mathrm{PCCl}$ & Ignição por Compressão de Carga Pré-misturada & {$[-]$} \\
\hline $\mathrm{PCl}$ & Combustão de Ignição por Compressão com & \\
\hline & Carga Pré-misturada & {$[-]$} \\
\hline PDTB & Peroxido de di-terc-butílo & {$[-]$} \\
\hline PFI & Injeção de combustível no pórtico & {$[-]$} \\
\hline PEG & Polietileno glicol & {$[-]$} \\
\hline PMI & ponto morto inferior & 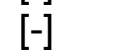 \\
\hline PMS & Ponto morto superior & {$[-]$} \\
\hline PME & Pressão Média Efetiva & [MPa] \\
\hline PREDIC & Combustão Diesel Pré-misturada de Baixa Carga & \\
\hline ppm & Partes por Milhão & \\
\hline Qa & Calor Aparente & {$[\mathrm{J}]$} \\
\hline QD & Carga de óleo diesel & [g] \\
\hline QE & Carga de etanol & \\
\hline $\mathrm{RCCl}$ & Ignição por compressão de reatividade controlada & \\
\hline RPM & Rotações Por Minuto & [IIIII] ] \\
\hline
\end{tabular}




$\begin{array}{lll}\text { RPS } & \text { Rotações por segundo } & {\left[\mathrm{s}^{-1}\right]} \\ \text { SAE } & \text { Sociedade de Engenheiros Automotivos } & {[-]} \\ \text { SCR } & \text { Catalizador de Redução Seletiva } & {[-]} \\ \text { Sol } & \text { Início da Injeção } & {[-]} \\ \text { S } & \text { Curso do pistão } & {[\mathrm{mm}]} \\ \text { T } & \text { Temperatura } & {\left[{ }^{\circ} \mathrm{C}, \mathrm{K}\right]} \\ \text { TGV } & \text { Turbina de Geometria Variável } & {[-]} \\ \text { TP } & \text { Tempo entre Pulsos } & {[\mathrm{ms}]} \\ \text { TS } & \text { Taxa de Substituição } & {[-]} \\ \text { TC } & \text { Taxa de Compressão } & {[-]} \\ \text { UCE } & \text { Unidade de Controle Eletrônica } & {[-]} \\ \text { UMF } & \text { Unidade para medição de fumaça } & {[-]} \\ \text { Up } & \text { Velocidade média do pistão } & {[\mathrm{mm} / \mathrm{s}]}\end{array}$

\section{Subscritos e Letras Gregas}

$\begin{array}{lll}\curlyvee & \text { Expoente politrópico } & {[-]} \\ \Delta T & \text { Diferença de temperatura } & {\left[{ }^{\circ} \mathrm{C} ; \mathrm{K}\right]} \\ \mu & \text { Viscosidade } & {\left[\mathrm{N} \mathrm{s} \mathrm{m}^{-2}\right]} \\ \rho & \text { Densidade ou massa especifica } & {\left[\mathrm{kg} / \mathrm{m}^{3}\right]} \\ \phi & \text { Razão de equivalência } & {[-]} \\ r & \text { real } & {[-]} \\ \mathrm{e} & \text { estequiométrica, etanol } & {[-]} \\ \mathrm{d} & \text { diesel } & {[-]} \\ \text { inj } & \text { relativo à injeção } & {[-]} \\ \text { rail } & \text { relativo ao rail de pressão } & {[-]} \\ \text { max } & \text { máximo } & {[-]} \\ \text { a } & \text { ar } & {[-]} \\ \mathrm{c} & \text { combustível } & {[-]}\end{array}$

